\title{
Friedreich ataxia
}

This patient describes his experience of Friedreich ataxia, from diagnosis at 14 years old to being confined to a wheelchair for nearly 30 years and developing serious problems with speech and swallowing.

\author{
Peter Gibilisco research fellow, patient ${ }^{1}$, Adam P Vogel senior research fellow ${ }^{2}$
}

${ }^{1}$ Social and Political Sciences, The University of Melbourne, Australia; ${ }^{2}$ Friedreich Ataxia Clinic, Monash Medical Centre, Melbourne, Australia, and Speech Neuroscience Unit, The University of Melbourne, Australia

This is one of a series of occasional articles by patients about their experiences that offer lessons to doctors.

I have had to struggle with a disability and consequent socioeconomic obstacles since a teenager. I was 14 years old when I was diagnosed with Friedreich ataxia, a progressive disease causing impairment to the nerves and so a failure of timely muscle reactions throughout my body. My only apparent initial physical abnormalities were the appearance and uncoordinated gait of someone under the influence of alcohol, but I quickly became expert at hiding such frailties. For whatever reason, I was never without friends, and was never, to my knowledge, made fun of by school friends and peers.

When I was 18, my mother died of cancer. This was an emotional time, and my disease temporarily took control of me, leading to a physical and emotional downward spiral.

Fortunately, the closeness of family and friends helped ease the pain. They helped me attain some form of self esteem, and to maintain pride in my life. At $18 \mathrm{I}$ was free and able to seek activities and forms of stimulation that were outside the regular happenings of adolescence. I succumbed to the lure of city based discothèques, where not everybody appreciated my uniquely uncoordinated style of dancing. I was an easy target for bullies, and on a couple of occasions this developed into trouble.

Muscular growth was hampered, giving rise to severe deformities, limitations, and other problems. For example, I have had to deal with severe scoliosis and cardiomyopathy. By 23 , I was reliant on a wheelchair, but now I'm simply too uncoordinated to make use of an electric one. As you may realise, the condition also leads to severely slurred speech, which, by the time I was 40 years old, meant my communication was also seriously impaired. When I finished my PhD, there was a demand for speaking at conferences, but I never could. I used to have someone else read out my papers. It was hard for me to socialise and make connections within academia because of my slurred speech. This also resulted in people thinking that I had a cognitive disability, and hence treating me in that way. Therefore, I have to rely heavily on typing, but this is difficult due to my typing speed of 1-2 words per minute. I am also having a lot of trouble recently with my vision as it is becoming harder for me to focus on certain objects. Generally, it takes a while for me to focus on any particular object, and some conditions of light and glare make it even more difficult.

I have lived on my own for 21 years, and during that time I completed a double degree at Monash University, Australia, in arts and accounting, then a masters degree, followed by a $\mathrm{PhD}$ in sociology at the University of Melbourne. During my PhD, I received the June Opie Fellowship from Auckland University and a Melbourne research scholarship. Immediately after this, I was awarded the Emerging Disability Leader of the Year award from the Victorian Disability Professionals.

Each of the steps I have made through my higher education has deepened my desire to promote and harness the capabilities of people who, in diverse ways, are not diminished by disability. I am convinced that somehow we-all of us-need to find a way to view and support people with disabilities in proactive and caring ways, taking equal opportunity as a principle for a way of life that is flexible enough to assist diverse disablement in diverse ways. Rather than focusing on combating the uncaring and judgmental stereotypes that arise from the medical model's view of sympathetic charity, we need simply to face up to people with severe disabilities as people. That is, we need a flexible and empathetic approach, aligned to an appreciation of the diverse social abilities, responsibilities, and opportunities that become evident when people interact with each other as fellows, as equals.

After completing my postgraduate degrees, I still did not want to face the harsh stereotypical views of many in the world of employment, and the possibility of interviews with employers making decisions on the basis of stereotypes about people with 
severe physical disabilities. Such ridiculous stereotypes confuse the functions of those with severe physical problems with those of severe intellectual disability. And so people like me, with severe physical disabilities, are often believed to be intellectually disabled as well. It works the other way too. Such discriminatory misjudgments can be damaging, especially to people who have severe disabilities and already suffer from low self esteem.

The need for support for my impairment has placed me under constant pressure. That is, I want to be able to function and work at fulfilling my desired needs and wants, but this requires appropriate hours of attendant care to function. Unfortunately, I am not independently wealthy, and the state provided system does not provide, in its current form, all that is required for adequate care.

For example, during the past 20 years of living independently and alone with a degenerative disability, I have had the constant need for the additional support of a personal carer. This provision of care, however, has not been responsive to my changing needs and life circumstances, preventing me from achieving a rich and fulfilling life. In Australia, part of the state disability plan focuses on supporting people with a disability in flexible ways, based on their individual needs, so that each person can live the lifestyle they want to lead. Regrettably, I don't feel the plan reaches its goal.

After my PhD, my options have improved, with my winning of the Disability Professionals "Emerging Leader of the Year" in March 2007. But, in reality, were the cost-benefits involved in pursuing academic excellence all worth it? Will this give me the necessary backing to pursue my goals of assisting in the provision of a just and inclusive society for people with disabilities? Are such pursuits beyond me because of my affliction, or do my personal narratives identify the political hurdles with greater clarity for people with severe physical disabilities?

Competing interests: We have read and understood the BMJ Group policy on declaration of interests and have no relevant interests to declare.

Provenance and peer review: Not commissioned; not externally peer reviewed.

Accepted: 22 August 2013

Cite this as: BMJ 2013;347:f7062

(c) BMJ Publishing Group Ltd 2013 


\section{A clinician's perspective}

Friedreich ataxia is a multisystem neurodegenerative disorder and the most common of the hereditary ataxia syndromes, with a prevalence of $0.5-3 / 100000$ individuals of western European lineage. It is caused by a triplet repeat expansion in the FXN gene, which leads to reduced frataxin, a mitochondrial protein important for iron metabolism. There is currently no treatment proven to alter its natural course. Symptoms typically present at 10-15 years of age but can be earlier or considerably later, and patients are usually wheelchair dependent within 10-15 years of disease onset. Other deficits include dysarthria and dysphagia, progressive limb and gait ataxia, optic and auditory neuropathy, scoliosis, and cardiomyopathy. On the whole, cognitive function is preserved.

Peter raised several important issues relating to the progression and resultant management of symptoms in Friedreich ataxia. He has been dealing with the consequences of his disease for three decades. In line with the clinical trajectory of Friedreich ataxia, Peter was confronted with mobility issues early on. The severity of his ataxia meant that he was forced to use a wheelchair, but his situation changed when the loss of fine motor control meant that he could no longer use an electric wheelchair. Beyond the control of a chair, a clear theme of Peter's journey is the change is his ability to communicate and socialise with peers. Peter has severe dysarthria, and this has affected his work and social life. He has had to forgo many professional opportunities because of his speech difficulties. Peter also reports a feeling of isolation resulting from misattribution of cognitive impairment by others based on how he sounds and what he looks like. Individuals with Friedreich ataxia do develop severe physical impairments, yet their cognitive capacity is preserved. This dichotomy places individuals with Friedreich ataxia at risk of discrimination, leading to further discord.

Alternative and augmentative communication devices (such as text or picture to voice tools, or speech to text tools) are often recommended to supplement the "lost speech" of those with severe dysarthria, but their effectiveness is limited with Friedreich ataxia. The visual symptoms described by Peter are the manifestation of fixation abnormalities. Combined with limb ataxia and severe dysarthria, these visual disturbances make the use of alternative communication modes an arduous or often impossible task.

Through his professional and personal endeavours, Peter has strived to promote the capabilities of people who are affected by disability in different ways. In conditions where physical impairments inhibit the experience of an individual, it is important for clinicians and public to recognise the underlying potential of the person, irrespective of symptoms.

Adam P Vogel

\section{Resources for patients and health professionals}

Bidichandani SI, Delatycki MB. GeneReviews: Friedreich ataxia. Revised. University of Washington, 2012. (www.ncbi.nlm.nih.gov/books/ NBK1281/).

Friedreich's Ataxia Research Alliance (www.curefa.org/index.html). US charity that provides education, resources, and support to people with Friedreich ataxia.

Ataxia UK (www.ataxia.org.uk/). UK charity with the goal of finding a cure for a range of ataxias; it funds research into developing safe, effective ataxia treatments.

Friedreich Ataxia Research Association (Australasia) (www.fara.org.au/). FARA Australasia and the US Friedreich's Ataxia Research Alliance have co-funded research projects including the Collaborative Clinical Research Network $n$ Friedreich's Ataxia.

Clinical Care Guidelines are in preparation and will be available via www.curefa.org 